\title{
NUMERICAL SIMULATION OF THE FLOW AROUND A SQUARE CYLINDER USING THE VORTEX METHOD
}

\author{
V. G. Guedes a, \\ G. C. R. Bodstein ${ }^{\text {, }}$ \\ and M. H. Hirata ${ }^{c}$ \\ ${ }^{a}$ Centro de Pesquisas de Energia Elétrica \\ Departamento de Tecnologias Especiais \\ CP. 68007 CEP: $21944-970$ \\ Rio de Janeiro, RJ, Brasil \\ vanessag@cepel.br

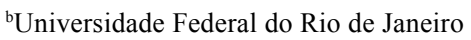 \\ Departamento de Engenharia Mecânica \\ CP. 68503 CEP: 21945-970 \\ Rio de Janeiro, RJ, Brasil \\ 'Universidade Federal de Itajubá \\ Departamento de Mecânica \\ Instituto de Engenharia Mecânica - CP. 50 \\ CEP: 37500-000 Itajubá, MG, Brasil
}

\begin{abstract}
The study of external incompressible flows around bluff bodies finds extensive applicability to real-life problems. Such flows are characterized by unsteady flow separation for high values of the Reynolds number, where a Von Karman-type periodic wake is formed. The prediction of these flows is very difficult, and one has usually to rely on specific experimental data to calculate the aerodynamic forces on the body. In order to numerically simulate this flow, this paper uses a new mesh-free two-dimensional Discrete Vortex Method associated with a Panel Method to calculate the lift and drag coefficients, as well as the pressure coefficient on a square cylinder, for a high Reynolds number flow. Lamb vortices are generated along the cylinder surface, whose strengths are determined to ensure that the no-slip condition is satisfied and that circulation is conserved. The impermeability condition is imposed through a source panel method, so that mass conservation is explicitly enforced. The dynamics of the body wake is computed using the convection-diffusion splitting algorithm, where the diffusion process is simulated using the random walk method, and the convection process is carried out with a lagrangian second-order time-marching scheme. Results for the aerodynamic forces and pressure distribution are presented.
\end{abstract}

Keywords: Vortex methods, panel method, high Reynolds number, aerodynamical forces.

\section{INTRODUCTION}

The study of external incompressible flows at high Reynolds numbers around bluff bodies finds extensive applicability to real-life problems, in addition to the recently renewed scientific interest as a means for testing numerical algorithms. Such flows are characterized by several different regimes that depend on the value of the Reynolds number, ranging from steady Stokes-type flows to strongly unsteady turbulent flows. For a wide range of Reynolds numbers a Von Karman-type periodic wake is formed. In most cases the occurrence of separation makes the prediction of these flows very difficult, and one has to rely on specific experimental data to calculate the aerodynamic forces on the body. Many attempts to numerically simulate most of the flow details have been reported in the literature, and a variety of both mesh-based and mesh-free methods have been used (Fusen and $\mathrm{Su}, 1998$; Taylor and Vezza, 1999).

In this work, we use the Vortex Method associated to the Panel Method to simulate the flow past a square cylinder. The flow field is calculated as the summation of a uniform flow, a cloud of vortices that model the boundary layer and wake vorticity in a lagrangian manner, and a series of potential flow singularities (sources in our case) distributed on panels arranged along the body surface. The panel distribution forms a closed polygon with a shape that approximates, as nearly as possible, the actual shape of the bluff body. The panel method is a technique intended to solve potential flow over 2-D and 3-D geometries, where the governing Laplace's equation is recast into an integral equation. The body surface is divided into panels or "boundary elements", and the integral is approximated by an algebraic expression on each of these panels (Moran, 1984). A system of linear algebraic equations is obtained for the unknown singularity strengths on the solid surface, which may be solved using techniques such as Gaussian elimination.

In our mesh-free vortex method, Lamb vortices are generated along the cylinder surface, whose strengths are determined to ensure that the no-slip condition is satisfied and that circulation is conserved. The impermeability condition is imposed through the application of a source panel method, so that mass conservation is explicitly enforced. The dynamics of the body wake is computed using the convection-diffusion splitting algorithm, where the diffusion process is simulated using the random walk method, and the convection process is carried out with a lagrangian secondorder time-marching scheme. We calculate global (e.g. lift and drag coefficients) as well as local (e.g. pressure coefficient) quantities for a high Reynolds 


\section{CIÊNCIA/SCIENCE}

number flow around a square cylinder. Results for the aerodynamic forces and pressure distribution are presented and compared to other results available in the literature.

\section{Mathematical Formulation}

We begin our analysis considering the flow around a square cylinder of side a, immersed in an unbounded region with a uniform flow and freestream speed U (Fig. 1). We assume the flow to be incompressible and two-dimensional, and the fluid to be newtonian with constant kinematic viscosity $v$. The unsteady flow that develops originates from the separation that occurs at the sharp corners of the cylinder surface, which generates an oscillatory wake downstream of the body. This flow is governed by the continuity and the Navier-Stokes equations, which can be written in the form

$$
\begin{aligned}
& \nabla \cdot \mathbf{u}=0, \\
& \frac{\partial \mathbf{u}}{\partial \mathrm{t}}+\mathbf{u} \cdot \nabla \mathbf{u}=-\nabla \mathrm{p}+\frac{1}{\operatorname{Re}} \nabla^{2} \mathbf{u} .
\end{aligned}
$$

In the equations above $\mathbf{u}$ is the velocity vector field, $\mathrm{p}$ is the pressure, and $\mathrm{Re} \equiv \mathrm{aU} / \mathrm{v}$ is the Reynolds number based on the square cylinder length side a. All the quantities in Eqs. (1), (2) and the equations below are nondimensionalized by $\mathrm{U}$ and $\mathrm{a}$.

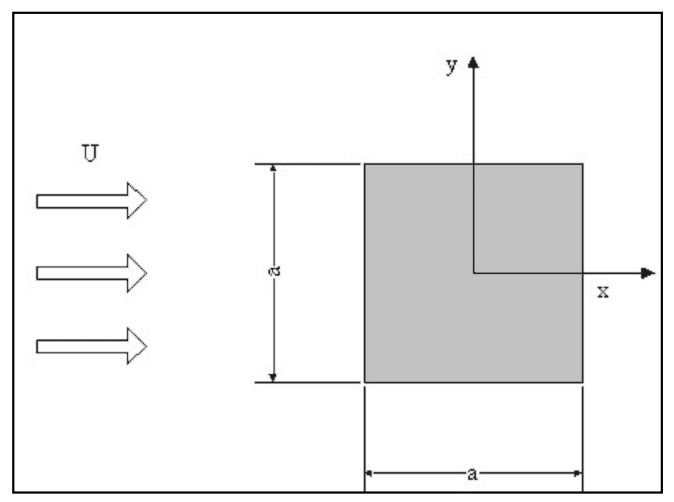

Figure 1. Flow around a square cylinder.

For all the cases studied, the flow is started impulsively from rest. The impermeability and the no-slip boundary conditions on the surface of the cylinder can be expressed as

$$
\begin{aligned}
& u_{n} \equiv \mathbf{u} \cdot \mathbf{n}=0, \text { on the cylinder surface } \\
& u_{t} \equiv \mathbf{u} \cdot \mathbf{t}=0, \text { on the cylinder surface }
\end{aligned}
$$

where $\mathrm{n}$ and $\mathrm{t}$ are unit vectors normal and tangential to the cylinder surface, respectively. We also require that

$$
|\mathbf{u}| \rightarrow 1 \text {, at infinity }
$$

The dynamics of the fluid motion, governed by the boundary-value problem (1)-(5), can be studied in a more convenient way if we take the curl of Eq. (2) and use Eq. (1) to obtain the vorticity equation. For a 2-D flow this equation is scalar, and it can be written as

$$
\frac{\partial \omega}{\partial \mathrm{t}}+\mathbf{u} \cdot \nabla \omega=\frac{1}{\operatorname{Re}} \nabla^{2} \omega
$$

where $\omega$ is the only non-zero component of the vorticity vector (in a direction normal to the plane of the flow).

In our model, the flow vorticity is represented by a cloud of $\mathrm{N}_{\mathrm{v}}$ discrete point vortices, each of constant strength $\Gamma_{\mathrm{j}}$. Using the source panel method (Anderson Jr., 1991), we can superimpose the flows comprised of the vortex cloud, the uniform flow and the flow due to a source distribution on the $\mathrm{N}$ panels, each of constant strength per unit panel length $\lambda_{j}$, to construct a flow field that satisfies Eqs. (1), (3) and (5) automatically. Figure2 shows the combined flow without the vortex cloud. Thus, the $\mathrm{u}$ and $\mathrm{v}$ velocities components of the total flow can be written in terms of the unknown vortex and source strengths and the known panel geometry according to the following equations

$$
\begin{aligned}
& \mathrm{u}(\mathrm{x}, \mathrm{y})=\sum_{\mathrm{j}=1}^{\mathrm{N}} \frac{\lambda_{\mathrm{j}}}{2 \pi}\left(\frac{\mathrm{C}_{\mathrm{u}}}{2} \ln \left(\frac{\mathrm{Sp}_{\mathrm{j}}^{2}+2 \mathrm{ASp}_{\mathrm{j}}+\mathrm{B}}{\mathrm{B}}\right)+\right. \\
& \left.+\frac{\mathrm{D}_{\mathrm{u}}-\mathrm{AC}_{\mathrm{u}}}{\mathrm{E}}\left(\tan ^{-1} \frac{\mathrm{Sp}_{\mathrm{j}}+\mathrm{A}}{\mathrm{E}}-\tan ^{-1} \frac{\mathrm{A}}{\mathrm{E}}\right)\right)+ \\
& -\frac{1}{2 \pi} \sum_{j=1}^{N_{v}} \Gamma_{j} \frac{\left(y-y_{j}\right)}{\left(x-x_{j}\right)^{2}+\left(y-y_{j}\right)^{2}} \\
& \mathrm{v}(\mathrm{x}, \mathrm{y})=\sum_{\mathrm{j}=1}^{\mathrm{N}} \frac{\lambda_{\mathrm{j}}}{2 \pi}\left(\frac{\mathrm{C}_{\mathrm{v}}}{2} \ln \left(\frac{\mathrm{Sp}_{\mathrm{j}}^{2}+2 \mathrm{ASp} \mathrm{p}_{\mathrm{j}}+\mathrm{B}}{\mathrm{B}}\right)+\right. \\
& \left.+\frac{D_{v}-A_{C_{v}}}{E}\left(\tan ^{-1} \frac{S_{j}+A}{E}-\tan ^{-1} \frac{A}{E}\right)\right)+ \\
& +\frac{1}{2 \pi} \sum_{j=1}^{N_{v}} \Gamma_{j} \frac{\left(x-x_{j}\right)}{\left(x-x_{j}\right)^{2}+\left(y-y_{j}\right)^{2}}
\end{aligned}
$$

where the constants above can be expressed in terms of the geometrical panel parameters, shown in Fig.3, as 


\section{CIÊNCIA/SCIENCE}

$$
\begin{aligned}
& \mathrm{A}=-\left(\mathrm{x}-\mathrm{X} \mathrm{p}_{\mathrm{j}}\right) \cos \varphi_{\mathrm{j}}-\left(\mathrm{y}-\mathrm{Y} \mathrm{p}_{\mathrm{j}}\right) \sin \varphi_{\mathrm{j}} \\
& \mathrm{B}=\left(\mathrm{x}-\mathrm{X} \mathrm{p}_{\mathrm{j}}\right)^{2}+\left(\mathrm{y}-\mathrm{Yp}_{\mathrm{j}}\right)^{2} \\
& \mathrm{C}_{\mathrm{u}}=-\cos \left(\varphi_{\mathrm{j}}\right) \\
& \mathrm{D}_{\mathrm{u}}=\mathrm{x}-\mathrm{Xp_{j }} \\
& \mathrm{Sp}_{\mathrm{j}}=\sqrt{\left(\mathrm{Xp}_{\mathrm{j}+1}-\mathrm{Xp} \mathrm{p}_{\mathrm{j}}\right)^{2}+\left(\mathrm{Yp}_{\mathrm{j}+1}-\mathrm{Yp} \mathrm{p}_{\mathrm{j}}\right)^{2}} \\
& \mathrm{E}=\sqrt{\mathrm{B}-\mathrm{A}^{2}}=\left(\mathrm{x}-\mathrm{X} \mathrm{p}_{\mathrm{j}}\right) \sin \varphi_{\mathrm{j}}-\left(\mathrm{y}-\mathrm{Yp} \mathrm{p}_{\mathrm{j}}\right) \cos \varphi_{\mathrm{j}} \\
& \mathrm{C}_{\mathrm{v}}=-\sin \left(\varphi_{\mathrm{j}}\right) \\
& \mathrm{D}_{\mathrm{v}}=\mathrm{y}-\mathrm{Yp_{j }}
\end{aligned}
$$

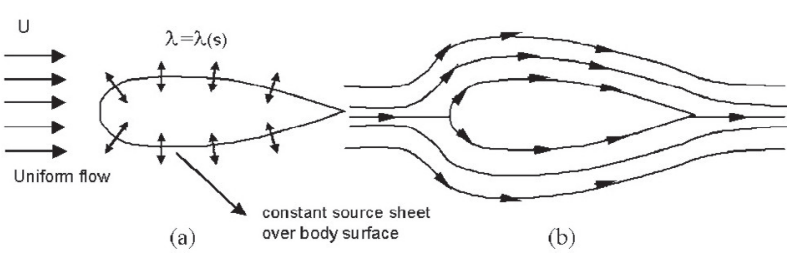

Figure 2. Uniform flow $U$ and constant source strength distribution $(\lambda)$ combined to construct a flow field around a arbitrary shaped body.

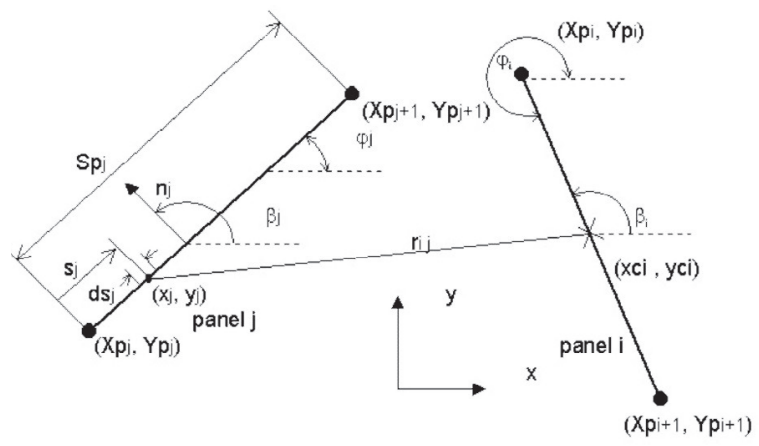

Figure 3. Geometry of the panels.

The aerodynamic force coefficients are calculated through integration of the pressure coefficient distribution around the square cylinder, which takes the form

$$
C_{D}=\int_{0}^{4 a} C_{p} \sin \varphi(s) d s
$$

and

$$
\mathrm{C}_{\mathrm{L}}=\int_{0}^{4 \mathrm{a}} \mathrm{C}_{\mathrm{p}} \cos \varphi(\mathrm{s}) \mathrm{ds}
$$

V. G. Guedes et al. Numerical Simulation of the Flow...

where $\mathrm{C}_{\mathrm{D}}$ and $\mathrm{C}_{\mathrm{L}}$ are the drag and lift coefficients, respectively. In order to calculate the pressure coefficient, we develop a new algorithm, based on the algorithm proposed by Fusen and $\mathrm{Su}(1998)$, such that $\mathrm{C}_{\mathrm{p}}$ at a panel control point $\mathrm{m}$ is calculated according to the following expression

$$
C_{P}=1+2\left(\sum_{n=1}^{m}\left(\frac{\Gamma_{n}}{\Delta t}-\Delta u_{n} \overline{u_{n}}\right)-\sum_{n=1}^{n_{\max }}\left(\frac{\Gamma_{n}}{\Delta t}-\Delta u_{n} \overline{u_{n}}\right)\right)
$$

where

$$
\Delta \mathrm{u}_{\mathrm{n}}=\left(\mathrm{u}_{(+)}-\mathrm{u}_{(-)}\right)
$$

and

$$
\overline{\mathrm{u}_{\mathrm{n}}}=\left(\frac{\mathrm{u}_{(+)}+\mathrm{u}_{(-)}}{2}\right) .
$$

The velocities $\mathrm{u}_{(+)}$and $\mathrm{u}_{(-)}$are calculated at the upper corners of a control volume bounded by panel $\mathrm{n}$ and enclosing a nascent vortex adjacent to the panel, as shown in Figure 4.

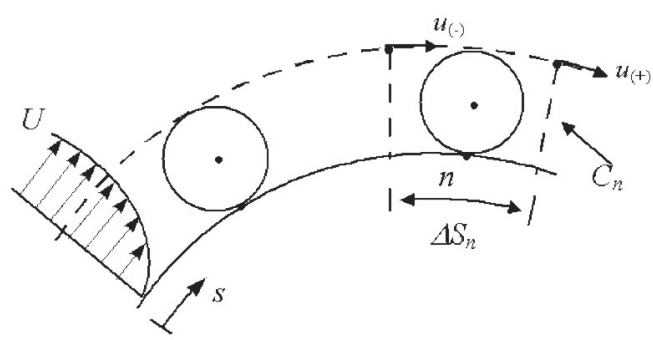

Figure 4. Tangential velocities at the upper corners of a control volume bounded by a panel and enclosing a nascent vortex adjacent to the panel.

\section{THE VORTEX METHOD - THE ALGORITHM}

The two-dimensional, incompressible, unsteady flow around a square cylinder formulated above is solved using a Discrete Vortex Method associated to a Source Panel Method. The Discrete Vortex Method uses an algorithm that splits the convective-diffusive operator (Chorin, 1973) in the form 


$$
\frac{\partial \omega}{\partial \mathrm{t}}=\frac{1}{\operatorname{Re}} \nabla^{2} \omega
$$

In a real flow vorticity is generated on the body surface so as to satisfy the no-slip condition, Eq. (4), and is transported by convection and diffusion into the flow according to Eq. (6). Our discrete vortex method represents the vorticity by discrete vortices, whose transport by convection and diffusion is carried out in a sequence within the same time step. First, a lagrangian approach is used to simulate the convective process, governed by Eq. (14). The convective motion of each vortex is determined by integration of each vortex path equation, which can be written, using a secondorder Adams-Bashforth scheme, as

$$
\begin{aligned}
& \Delta \mathrm{x}_{\mathrm{c}}=\left[\frac{3}{2} \mathrm{u}(\mathrm{t})-\frac{1}{2} \mathrm{u}(\mathrm{t}-\Delta \mathrm{t})\right] \Delta \mathrm{t} \\
& \Delta \mathrm{y}_{\mathrm{c}}=\left[\frac{3}{2} \mathrm{v}(\mathrm{t})-\frac{1}{2} \mathrm{v}(\mathrm{t}-\Delta \mathrm{t})\right] \Delta \mathrm{t}
\end{aligned}
$$

In Eqs. (16) and (17), $\Delta \mathrm{x}_{\mathrm{c}}$ and $\Delta \mathrm{y}_{\mathrm{c}}$ are displacements of a vortex owing to convection, and $\mathrm{u}$ and $\mathrm{v}$ are components of the velocity at the point occupied by the vortex. Second, the process of viscous diffusion, governed by Eq. (15), is simulated using the Random Walk Method (Lewis, 1991), where the random displacements of each vortex in the $\mathrm{x}$ and $\mathrm{y}$ directions owing to diffusion, $\Delta \mathrm{x}_{\mathrm{d}}$ and $\Delta \mathrm{y}_{\mathrm{d}}$, are calculated from

$$
\Delta \mathrm{x}_{\mathrm{d}}=\Delta \mathrm{r} \cos (\Delta \theta) \text { and } \Delta \mathrm{y}_{\mathrm{d}}=\Delta \mathrm{r} \sin (\Delta \theta)
$$

where

$$
\Delta \mathrm{r}=\left[8 \mathrm{Re}^{-1} \Delta \mathrm{t} \ln (1 / \mathrm{P})\right]^{1 / 2}, \text { and } \Delta \theta=2 \pi \mathrm{Q}
$$

In Eqs. (19), $P$ and $Q$ are random numbers between 0 and 1 , drawn from a uniform probability density distribution.

In order to remove the singularity of the point vortices we use Lamb vortices for $r \leq \sigma_{o}$, where $\sigma_{\mathrm{o}}$ is the radius of the vortex core. During a time step $\Delta$ t, the core grows from zero to $\sigma_{\mathrm{o}}$, where

$$
\sigma_{\mathrm{o}}=4.48364 \sqrt{\frac{\Delta \mathrm{t}}{\operatorname{Re}}}
$$

This value is kept constant for the entire simulation. In terms of $\sigma_{\mathrm{o}}$, the dimensionless velocity induced by the $\mathrm{k}^{\text {th }}$-vortex in the circumferential direction, $\mathrm{u}_{\theta_{\mathrm{k}}}$, is

$$
\mathrm{u}_{\theta \mathrm{k}}=\Gamma_{\mathrm{k}} / 2 \pi \mathrm{r}\left\{1-\exp \left[-\mathrm{C}\left(\mathrm{r}^{2} / \sigma_{\mathrm{o}}^{2}\right)\right]\right\}
$$

In this particular equation $r$ is the radial distance between the vortex center and the point in the flow field where the induced velocity is calculated, and $\mathrm{C}=5.02572$ is a constant. The distance $\varepsilon$ off the cylinder surface where the new vortices are generated per time step (Fig. 5) is set equal to $\sigma_{\mathrm{o}}$ for all the cases studied. Vortices that penetrate the body are reflected back into the flow field.

The time step $\Delta \mathrm{t}$ is estimated from the convective length and velocity scales of the flow. For a velocity scale of order one and a length scale of order of $\Delta \mathrm{s}=4 \mathrm{a} / \mathrm{N}$ between two adjacent vortices generated at the surface, we can write

$$
\Delta \mathrm{t}=\frac{4 \mathrm{k}}{\mathrm{N}}, 0<\mathrm{k} \leq 1
$$

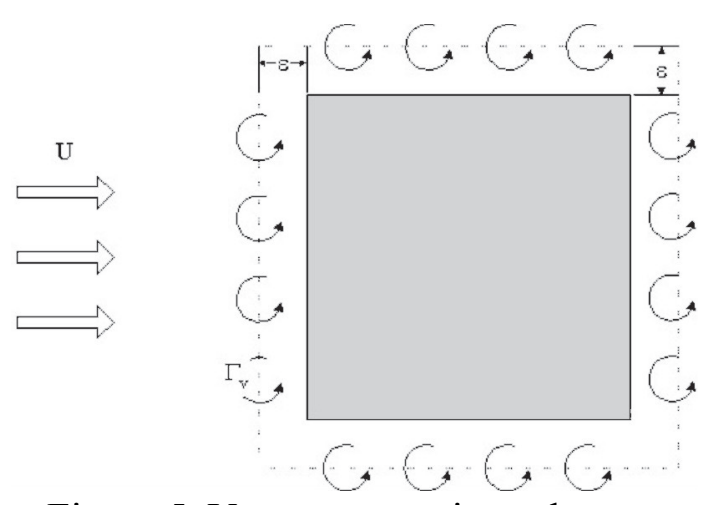

Figure 5. Vortex generation scheme.

\section{THE NUMERICAL IMPLEMENTATION}

Initially, the body surface is discretized into $\mathrm{N}$ uniform small panels. The numerical method described above is, then, implemented to run sequentially according to the following five steps: (i) generation of new vortices; (ii) calculation of the forces on the body; (iii) convection of the vortices; (iv) diffusion of the vortices; (v) reflection of some vortices; (vi) stepping in time.

The process of vorticity generation is carried out so as to satisfy the condition impermeability and the no-slip condition, Eqs. (3) and (4). At each time step, N new vortices are and 


\section{CIÊNCIA/SCIENCE}

(4). At each time step, $\mathrm{N}$ new vortices are created a small distance $\varepsilon$ off the body surface, just adjacent to the $\mathrm{N}$ panels (Fig. 5), and new $\mathrm{N}$ sources are created on the $\mathrm{N}$ panels that cover the body. The strengths of these new vortices and panel sources are determined by imposing the no-slip and the impermeability conditions at $\mathrm{N}-1$ control points on the cylinder surface, at the center of each panel. In order to implement the entire procedure, the velocities induced by all the vortices in the wake are computed at the $\mathrm{N}-1$ control points where Eqs. (3) and (4) must be satisfied. This contribution is added to the velocities induced by the new vortices and the source strengths and equated to zero. Thus, $2 \mathrm{~N}-2$ equations can be written out for the $2 \mathrm{~N}$ unknowns ( $\mathrm{N}$ new vortices and $\mathrm{N}$ new source strengths). The two last equations are statements of conservation of circulation (the sum of all vortices with known and unknown strengths must equal zero) and mass conservation (the sum of all new source strengths must equal zero). This procedure yields an algebraic system of $2 \mathrm{~N}$ equations and $2 \mathrm{~N}$ unknowns, that is,

$$
\begin{aligned}
& \sum_{k=1}^{N}\left(A_{j k} \Gamma_{k}(t)+B_{j k} \lambda_{k}(t)\right)=b_{j}(t), 1 \leq j \leq N-1 \\
& \sum_{k=1}^{N} \Gamma_{k}(t)=b_{N}(t), j=N \\
& \sum_{k=1}^{N}\left(C_{j k} \Gamma_{k}(t)+D_{j k} \lambda_{k}(t)\right)=b_{j}(t), 1 \leq j \leq N-1 \\
& \sum_{k=1}^{N} \lambda_{k}(t)=b_{N}(t), j=N
\end{aligned}
$$

The $2 \mathrm{~N} \times 2 \mathrm{~N}$ matriz given by Eqs (23) to (26) takes the form of

$$
\mathbf{A}=\left[\begin{array}{cc}
A_{j k} & B_{j k} \\
1 & 0 \\
C_{j k} & D_{j k} \\
0 & 1
\end{array}\right]
$$

The $(\mathrm{N}-1) \times \mathrm{N}$ matrix $\mathrm{A}_{\mathrm{jk}}$ represents the coefficient matrix of the normal velocities induced by all the vortices, the $(\mathrm{N}-1) \times \mathrm{N}$ matrix $\mathrm{B}_{\mathrm{jk}}$ represents the coefficient matrix of the normal velocities induced by the source panels, the $(\mathrm{N}-$ 1) $\times \mathrm{N}$ matrix $\mathrm{C}_{\mathrm{ik}}$ represents the coefficient matrix of the tangential velocities induced by the vortices, and the $(\mathrm{N}-1) \times \mathrm{N}$ matrix $\mathrm{D}_{\mathrm{jk}}$ represents the coefficient matrix of the tangential velocities induced by the source panels. The second and fourth rows, given by Eqs. (24) and (26), represent the conservation of vorticity and the conservation of mass at the last panel, respectively. As a consequence, the no-slip and the impermeability conditions are not satisfied at the control point of the last panel .

The linear system of algebraic equations can be written in closed form as

$$
\left[\begin{array}{cc}
A_{j k} & B_{j k} \\
1 & 0 \\
C_{j k} & D_{j k} \\
0 & 1
\end{array}\right] \cdot\left[\begin{array}{l}
\Gamma_{k}(t) \\
\lambda_{k}(t)
\end{array}\right]=\left[b_{j}(t)\right]
$$

The elements of the $2 \mathrm{~N} \times 2 \mathrm{~N}$ matrix $\mathbf{A}$ depend on the position of the vortices just created and on the points on the cylinder surface where the no-slip and impermeability conditions are imposed (control points of the panels). It is, therefore, calculated only once for the entire simulation. The vector $b_{j}(t)$, which is recalculated every time step, includes the contribution of all the terms in Eqs. (7) and (8).

\section{RESULTS AND DISCUSSION}

We now present results for the simulation of a high Reynolds number, two-dimensional, incompressible, unsteady flow around a square cylinder. The surface of the square cylinder is discretized into 200 panels. A long-time simulation is performed up to $t=40.0$, where 200 vortices are generated per time step. Since the algorithm is still being tested, we present preliminary results for one case only, with $\operatorname{Re}=10^{5}$. The numerical parameters used in the computations are: $\Delta \mathrm{t}=0.1$ and $\varepsilon=\sigma_{\mathrm{o}}=$ 0.0045 . This run is carried out for 400 time steps and it ends up with 80000 vortices in the flow.

The flow around a circular cylinder presents several interesting characteristics, which can be described starting with the occurrence of the separation phenomenon. Experiments show the formation of the so-called Von Karman vortex street, which is comprised of large vortices generated and shed alternately from the upper and lower surfaces of the cylinder. The vortices in the wake are connected in pairs by a vortex sheet. Owing to the periodic characteristics of the wake, 
the lift force on the cylinder oscillates in time around zero, with a frequency determined by the Strouhal number. The periodic behavior of the wake is also reflected on the drag force, which presents a time evolution that oscillates around a non-zero mean value. However, its non-dimensional frequency is approximately twice the Strouhal number, since the drag force presents approximately two cycles of oscillation for each cycle of the lift force. For these reasons, the experimental values available in the literature represent time averages for the lift and drag coefficients.

Figure 6 illustrates the positions of the vortices present in the flow simulation at $\mathrm{t}=40.0$. The wake clearly shows the occurrence of separation at the corners of the square cylinder surface, followed by the generation and growth of large separated vortices near the lateral and the backward surfaces of the cylinder, which precedes their shedding into the wake. It can also be seen the formation of pairs of the large eddies that comprise the Von Karman vortex street, connected to each other by thin vortex sheets. The cores of these large vortices grow as they move downstream of the cylinder due to the diffusive effect of the flow, and the lateral extent of these vortices increase due to the convective growth of the instabilities in the wake. Note that the farthest vortices in the wake are generated at the initial moments of the simulation, and they are, therefore, subject to numerical transient effects.

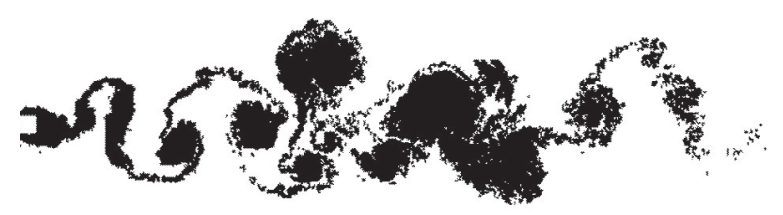

Figure 6. Positions of the wake vortices for $\mathrm{Re}=$ $10^{5}$, at $\mathrm{t}=40.0 ; \mathrm{N}=200, \Delta \mathrm{t}=0.1$ and $\varepsilon=\sigma_{\mathrm{o}}=$ 0.0045 .

The time histories of the lift and drag coefficients are revealed in the graph of Fig. 7. As expected these coefficients reach a periodic steady state after an initial numerical transient (about 20 units of non-dimensional time). The lift coefficient oscillates about zero with a non-dimensional frequency (Strouhal number, St) of 0.138. This value for $S t$ is calculated over two cycles of oscillation corresponding to the $\mathrm{C}_{\mathrm{L}}$ peaks at $\mathrm{t}=22.5$ and $t=37.0$. On the other hand, the drag force oscillates about a mean value of 1.88 , integrated over the same cycles used to determine St.

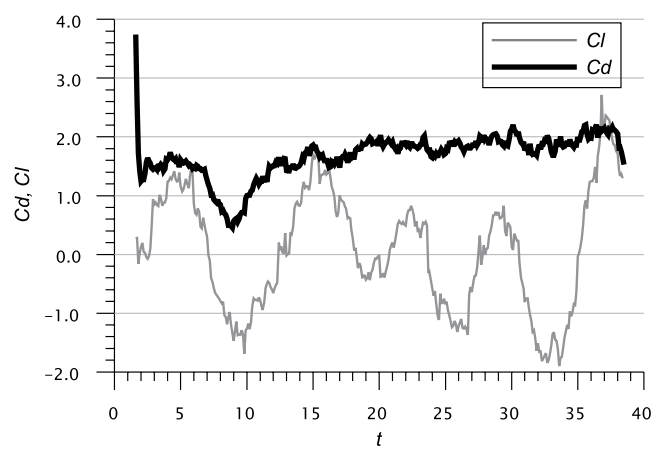

Figure 7. Time variation of $C_{D}$ and $C_{L}$ for $\mathrm{Re}=10^{5}$, at $\mathrm{t}=40.0 ; \mathrm{N}=200, \Delta \mathrm{t}=0.1$ and $\varepsilon=\sigma_{\mathrm{o}}=0.0045$.

The pressure coefficient is shown in Fig. 8, compared to the experimental results obtained by Lee (1975) for $\mathrm{Re}=1.76 \times 10^{5}$. As mentioned above, the numerical scheme used to compute the pressure coefficient distribution is based on the model proposed by Fusen and Su (1998). Our new scheme is observed to perform better than the scheme used by Mustto et al. (1998) for a circular cylinder, which is based on Lewis (1991). Our numerical results compare well with the experimental results of Lee (1975), although the calculated backpressure distribution is slightly higher than the experimental one. We should point out that the difference in the Reynolds number between the numerical and experimental results is also a source of discrepancy.

Table 1 provides an easy comparison of our numerical results for the drag coefficient and Strouhal number with the experimental results obtained by Blevins (1984) and Vickery (1966). The experimental mean drag coefficient of Blevins is 2.20 , which is $7.3 \%$ higher than the value 2.05 measured by Vickery (1966). Our calculation produces a mean drag coefficient of 1.88 , which is lower than both experimental values. This fact is clearly due to the higher backpressure calculated in the simulation. Our numerical value for the Strouhal number, 0.138 , is higher than the experimental ones shown in the Table, namely 0.12 and 0.118 , obtained by Blevins and Vickery, respectively. This difference may be attributed to errors in the simulation of the vortex shedding mechanism near the corners of the cylinder. Although close to the experimental values, the numerical results indicate that the algorithm still needs investigation in order to yield more accurate results. The numerical parameters also require further tests, searching for more adequate values. On the other hand, difficulties in calculating massively separated flows are expected if one recognizes that even experimental results present relatively large discrepancies when compared to each other. 


\section{CIÊNCIA/SCIENCE}

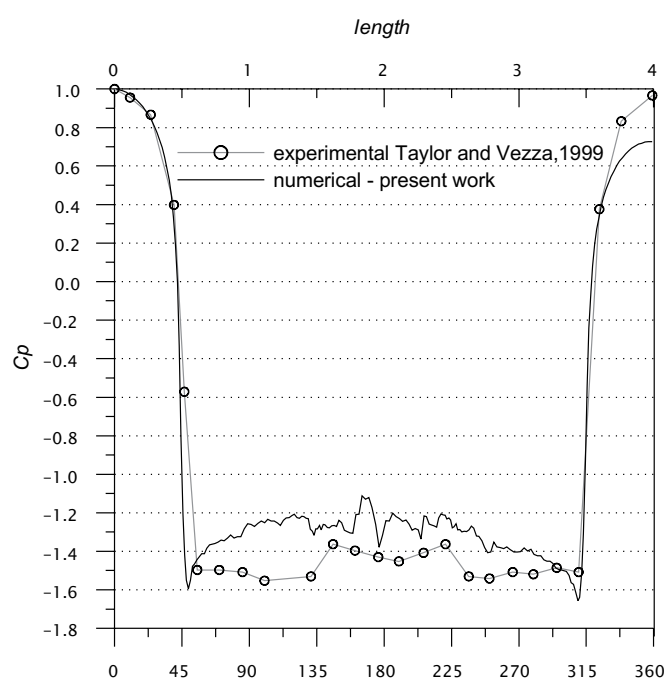

Figure 8. Cp distribution on the square cylinder surface at $\mathrm{t}=40.0$, for $\mathrm{Re}=10^{5}, \mathrm{~N}=200, \Delta \mathrm{t}=0.1$ and $\varepsilon=\sigma_{\mathrm{o}}=0.0045$.

Table 1. Comparison of the mean drag coefficient and Strouhal number with other numerical and experimental results, for $\mathrm{Re}=10^{5}$.

\begin{tabular}{|c|c|c|}
\hline Results & $\mathrm{C}_{\mathrm{D}}$ & $\mathrm{St}$ \\
\hline Blevins (1985): experimental & 2.20 & 0.120 \\
\hline Vickery (1966): experimental & 2.05 & 0.118 \\
\hline Present results: numerical & 1.88 & 0.138 \\
\hline
\end{tabular}

\section{CONCLUSIONS}

A new mesh-free two-dimensional discrete vortex method coupled with a source panel method is implemented to calculate the Strouhal number and the lift, drag and pressure coefficients on a square cylinder in a two-dimensional, incompressible, unsteady and high Reynolds number flow.

Considering that the simulation described in this paper is still preliminary, we find that the numerical results obtained are in good agreement with the experimental results used for comparison. The discrepancies observed in the determination of the Strouhal number and in the calculation of the backpressure distribution on the cylinder surface, which is transferred to the drag coefficient, may be attributed to errors in the simulation of the dynamics of the vortex shedding mechanism. Although the differences to the experimental values are not large, the numerical results suggest that the algorithm still needs investigation in order to yield more accurate results. An increase in the resolution of the simulation, through an increase of the number of vortices, may attenuate part of the
V. G. Guedes et al. Numerical Simulation of the Flow...

problem. The numerical parameters used also require further tests to find more adequate values. However, it must be pointed out that the numerical calculation of massively separated flow is expected to be difficult due to the flow complexity. This fact is corroborated by the relatively large discrepancies between experimental results available in the literature, when compared to each other. The use of a fast summation scheme to determine the vortex-induced velocities, such as the Multipole Expansion scheme, allows an increase in the number of vortices and a reduction of the time step, which increases the resolution of the simulation, in addition to a reduction of the CPU time, which allows a longer simulation time to be carried out. This will definitely improve the numerical results.

\section{REFERENCES}

Anderson, J. D., 1991. Fundamentals of Aerodynamics, 2nd. Edition, McGraw Hill.

Blevins, R. D., 1984, Applied Fluid Dynamics Handbook, Van Nostrand Reinhold Co.

Chorin, A. J., 1973, Numerical Study of Slightly Viscous Flow, Journal of Fluid Mechanics, Vol. 57, pp. 785-796.

Fusen H. E. e Tsung-Chow Su, 1998, A Numerical Study of Bluff Body Aerodynamics in High Reynolds Number Flows by Viscous Vortex Element Method, Journal of Wind Engineering and Industrial Aerodynamics, Vol. 77-78, pp. 393-407.

Lee, B. E., 1975, The Effect of Turbulence on the Surface Pressure Field of Square Prism, Journal of Fluid Mechanics, Vol. 69, Part 2, pp. 263-282.

Lewis, R. I., 1991, Vortex Element Methods for Fluid Dynamic Analysis of Engineering Systems, Cambridge University Press, Cambridge.

Moran, J., 1984. An Introduction to Theoretical and Computational Aerodynamics, John Wiley.

Mustto, A. A., Hirata, M. H., Bodstein, G. C. R., 1998, Discrete Vortex Method Simulation of the Flow around a Circular Cylinder with and without Rotation, AIAA Paper 98-2409, $16^{\text {th }}$. Applied Aerodynamics Conference, Albuquerque, N. M., June 15-18.

Taylor, I. and Vezza, M., 1999, Prediction of Unsteady Flow around Square and Rectangular Section Cylinders Using Discrete Vortex Method, Journal of Wind Engineering and Industrial Aerodynamics, Vol. 82, pp. 247-269.

Vickery, B. J., 1966, Fluctuating Lift and Drag on a Long Cylinder of Square Cross-section in a Smooth and in a Turbulent Stream, Journal Fluid Mechanics, Vol. 25, pp. 481-494. 